\title{
Laparoscopic resection of primary adrenal ganglioneuroma: A case report and review of the literature
}

\author{
YUAN ZHOU ${ }^{1}$, QILIAN LIANG ${ }^{1}$, WEN-TING OU ${ }^{1}$, ZHOU-YU LI $^{2}$ and QIU-LONG LIU ${ }^{1}$ \\ ${ }^{1}$ Department of Oncology, Affiliated Hospital of Guangdong Medical College, Zhanjiang, Guangdong 524001; \\ ${ }^{2}$ Department of Radiotherapy, Cancer Center of Guangzhou Medical University, Guangzhou, Guangdong 510095, P.R. China
}

Received June 5, 2014; Accepted February 10, 2015

DOI: 10.3892/ol.2015.3021

\begin{abstract}
Adrenal ganglioneuroma (AGN) is an extremely rare, benign tumor that originates from the neural crest tissue of the sympathetic nervous system. The majority of cases are detected incidentally, since the disease often lacks clear clinical manifestations or is asymptomatic. In addition, AGN is often misdiagnosed as being an adrenal adenoma or adrenal pheochromocytoma. The present study describes a 58-year-old female who visited the outpatient clinic of the Affiliated Hospital of Guangdong Medical College (Zhanjiang, Guangdong, China) with symptoms of face and lower extremity dropsy. Color Doppler ultrasonography revealed a solid tumor in the right kidney, and abdominal computed tomography identified an irregular, solid tumor measuring $\sim 6 \times 4.5 \times 7 \mathrm{~cm}^{3}$ and arising from the right adrenal gland, with a clear boundary. Magnetic resonance imaging was not performed. An initial diagnosis of adrenal adenoma was established. The patient was treated by laparoscopy in order to remove the tumor. However, following surgery, a pathological examination suggested that the tumor was a GN originating from the adrenal medulla. The formation of a correct diagnosis can be extremely challenging, as AGNs do not exhibit any specific clinical manifestations. Therefore, detection often depends entirely upon imaging studies, and the final diagnosis can be only by confirmed following a histopathological evaluation.
\end{abstract}

\section{Introduction}

Ganglioneuroma (GN) is a benign and slow-growing tumor that originates from the primitive neural crest cells. Histologically, the tumor is composed of Schwann mesenchymal cells and gangliocytes. GNs may arise in any region of the

Correspondence to: Professor Qilian Liang, Department of Oncology, Affiliated Hospital of Guangdong Medical College, 57 Renmin Avenue, Zhanjiang, Guangdong 524001, P.R. China E-mail: lianqilian@gdmc.edu.cn

Key words: neuroganglioma, laparoscopic adrenalectomy, adrenal tumor paravertebral sympathetic plexus (1-4), but are typically observed in the posterior mediastinum (39-43\%) or the retroperitoneum (32-52\%). By contrast, the neuromas rarely occur in the adrenal glands $(5,6)$. The majority of patients with adrenal GNs (AGNs) do not exhibit any clinical symptoms. Furthermore, the tumors are usually clinically dormant and asymptomatic, even when large in size (1). Following the extensive use of imaging procedures, such as ultrasonography and computed tomography (CT), the number of diagnosed GNs has increased $(6,7)$. A large number of AGNs are identified during physical examinations or due to the presence of other diseases. The incidence of AGN is gradually increasing in China, due to increased awareness with regard to early detection; and dozens of cases have been reported in recent years (8). The majority of previous studies concerning AGNs have been case reports, and information regarding the pathogenesis of the disease is limited. The establishment of a guideline for clinicians has proven extremely challenging, and therefore, AGNs are often misdiagnosed as a different type of tumor $(8,9)$. The present study describes a large AGN that was incidentally identified in a 58-year-old female. The diagnosis was confirmed by histopathological examination. Following diagnosis, the patient was treated by laparoscopy. The present study also includes a review of the relevant literature in order to provide clinicians with information concerning this uncommon malignancy. Written informed consent was obtained from the patient.

\section{Case report}

A 58-year-old female was admitted to the Department of Oncology at the Affiliated Hospital of Guangdong Medical College (Zhanjiang, Guangdong, China) in May 2011 complaining of face and lower extremity dropsy that had been apparent for $>20$ days. The patient had no symptoms of headaches, abdominal pain, nausea, vomiting, urinary urgency or fever, and there was no loss of appetite or weight. An ultrasound scan, which had been performed two days previously at a local hospital, had revealed a mass in the kidney. The patient's medical history was unremarkable. The patient's blood pressure was within the normal range and demonstrated no fluctuations. In addition, a physical examination did not reveal an abnormal mass, tenderness or rebound tenderness in the abdomen. 
Laboratory tests revealed a cortisol level of $6.78 \mu \mathrm{g} / \mathrm{dl}$ (normal range, $<10 \mu \mathrm{g} / \mathrm{dl}$ ) and an adrenocorticotrophin level of $<10 \mu \mathrm{g} / \mathrm{dl}$ (normal range, $<46 \mu \mathrm{g} / \mathrm{dl})$. Catecholamine metabolites in the blood and urine were within normal ranges. Furthermore, routine tumor markers demonstrated no abnormalities and the fecal occult blood test was negative.

Contrast-enhanced $\mathrm{CT}$ of the upper abdomen revealed an irregular and homogenous solid tumor in the right adrenal gland, measuring $\sim 6 \times 4.5 \times 7 \mathrm{~cm}^{3}$. The $\mathrm{CT}$ value was $\sim 28 \mathrm{HU}$. The arterial phase $\mathrm{CT}$ value was $\sim 31 \mathrm{HU}$ and the venous phase $\mathrm{CT}$ value was $50 \mathrm{HU}$. The lesion and surrounding structures exhibited clear demarcation. The left adrenal gland parenchyma was uniform in density and demonstrated enhanced evenness, and there was no evidence of abnormal nodes or an enhanced soft-tissue mass (Fig. 1). Retroperitoneal lymph node involvement was not detected, and there was no evidence of invasion to the adjacent tissues or organs. Chest X-ray did not reveal any abnormal lesions.

Due to the patient's physical condition, a laparoscopic complete excision of the mass was performed through a transabdominal approach. Following surgery, pathological and immunohistological examinations were performed. The cut surface of the tumor was solid, whitish-gray in color, and demonstrated no evidence of necrosis or hemorrhage. Microscopically, the sections were composed of spindle-shaped cells that demonstrated an irregular growth pattern and occasional scattered mature ganglionic cells with focal lymphocytic infiltration and dystrophic calcification (Fig. 2A and B). The immunohistochemical analysis revealed that the ganglion cells were positive for neuron-specific enolase (NSE; Fig. 3A), S-100 (Fig. 3B) and cluster of differentiation (CD)56 (Fig. 3C), but negative for cytokeratin (Fig. 3D), leukocyte common antigen (Fig. 3E) and smooth muscle actin (Fig. 3F). The specimen did not exhibit any histological evidence of malignant degeneration. These results established a histological diagnosis of AGN.

The post-operative period was uneventful, and the patient was discharged on the fifth day after surgery. The patient returned to the hospital 18 months later in good health. CT did not reveal any evidence of tumor recurrence or metastasis, and the cosmetic result was satisfactory.

\section{Discussion}

GN originates from primitive neural crest cells, arises in sympathetic ganglion cells and is composed of mature Schwann cells, nerve fibers and ganglion cells $(2,3,7,10)$. Tumors originating from ganglion cells form a family consisting of GNs, ganglioneuroblastomas and neuroblastomas. Ganglioneuroblastomas exhibit intermediate differentiation, whilst neuroblastomas are highly-malignant tumors (1-3). GNs most often occur in children and young adults aged between 10 and 40 years old $(9,11)$. AGN is an extremely rare, differentiated, benign and slow-growing tumor. In adults, it most commonly affects the right adrenal gland (6). The majority of GN patients do not exhibit any clinical manifestations. However, if they do, the most common one is an asymptomatic, slow-growing abdominal mass or abdominal pain. Due to its hormonally, non-secreting nature, an AGN may rarely cause symptoms of diarrhea, virilization and hypertension $(2,12-14)$. This phenotype is attributed to that fact that these tumors do not secrete excess catecholamines or steroid hormones (15). The majority of patients with GNs are hormonally silent. However, a minority of patients do produce hormones, such as catecholamines, vasointestinal peptide and androgens, which result in symptoms of weakness, diarrhea, hypertension and virilization $(13,14,16,17)$.

Upon CT, AGNs usually appear as oval or round, homogenous and well-circumscribed masses, which often partially or completely surround major blood vessels. AGNs develop as slightly hypodense masses, with multiple punctate calcifications visible internally. Enhanced CT scan of the tumor focus is without enhancement or with only mild enhancement $(2,18)$.

Microscopically, the tumor sections are primarily composed of spindle cells clustered into fascicles. Mature ganglion cells scattered or arranged in small clusters are also observed (1). A diagnosis of GN depends upon whether or not the tumor is composed of ganglion cells (19). Immunohistochemical results, including S100, CD56, synaptophysin and NSE positivity, are also important for forming a diagnosis (20).

Distinguishing between AGNs and other neurogenic tumors, such as neuroblastomas, ganglioneuroblastomas, pheochromocytomas and adenomas, can be challenging $(1,13)$. Neuroblastomas and ganglioneuroblastomas, which originate from ganglion cells, usually occur in younger patients and children. These tumors develop at the same sites as GNs, but are generally more aggressive and metastasize to the bone, liver and lung in $~ 50 \%$ of cases (21). Since pheochromocytomas secrete catecholamine or steroid hormones, patients often present with symptoms of paroxysmal hypertension associated with palpitations, sweating and dizziness or headaches (22). Patients also usually present with a family history of the disease $(23,24)$. However, since GNs generally lack clear clinical symptoms or are asymptomatic, patients with hypertension may require further hormonal investigations (25). Specific endocrine analyses should be performed as a definite differential approach in order to detect hormone levels. An elevated catecholamine level in a 24-h urine sample is suggestive of a pheochromocytoma. By contrast, the same test may be negative in patients with GNs, since these tumor cells do not secrete catecholamine hormones (15). CT and magnetic resonance imaging are commonly used during the formation of a differential diagnosis. Upon CT, AGNs appear as solid, round adrenal masses without evidence of tumor invasion. The most prominent imaging finding of an AGN is a count of $<40 \mathrm{HU}$, whereas a significant enhancement upon CT is commonly observed in adrenal pheochromocytomas (26). A pathological investigation is often a conclusive diagnostic determinant used to differentiate between these tumors. The gross and histopathological morphological appearance and immunohistochemical features differ significantly due to the different origins of the tumors (20).

Laparoscopic adrenalectomy has become the gold standard curative treatment for the majority of patients with adrenal tumors. Due to its minimally invasive nature, laparoscopy may a be more suitable approach to treat AGNs compared with traditional open abdominal surgery $(27,28)$. All AGN patients undergo an open or laparoscopic adrenalectomy, depending upon detailed analysis of individual criteria, taking into consideration the tumor location, function and 

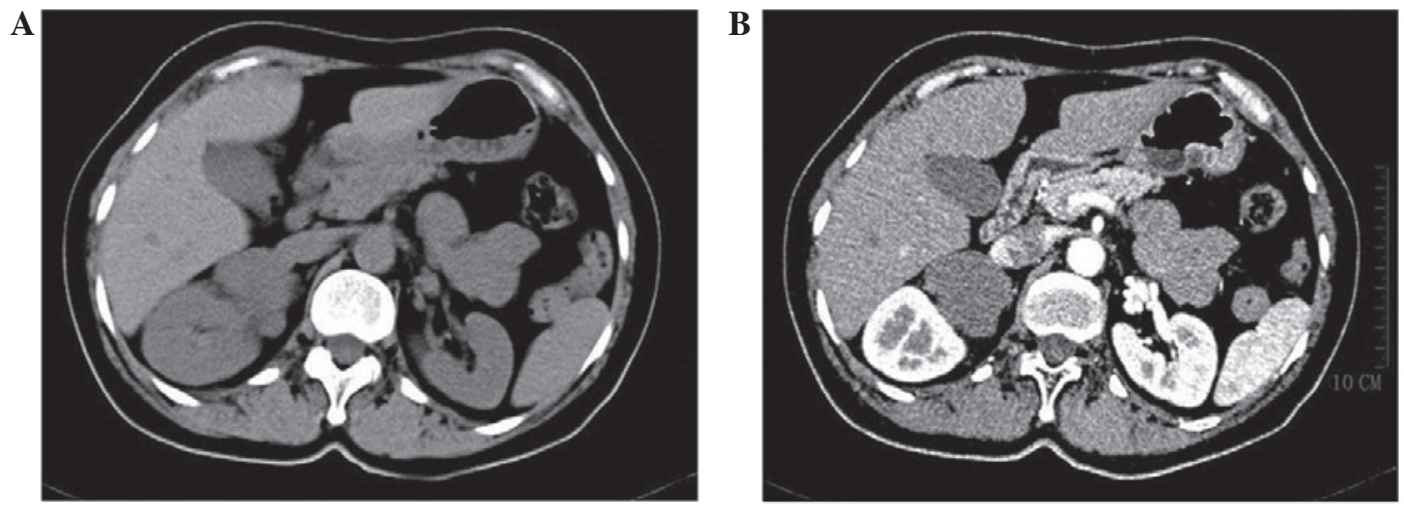

Figure 1. (A) Unenhanced CT revealing an irregular and homogenous solid tumor measuring $28 \mathrm{HU}$ in the right adrenal gland. (B) Enhanced CT revealing an enhanced mass measuring $50 \mathrm{HU}$. CT, computed tomography.
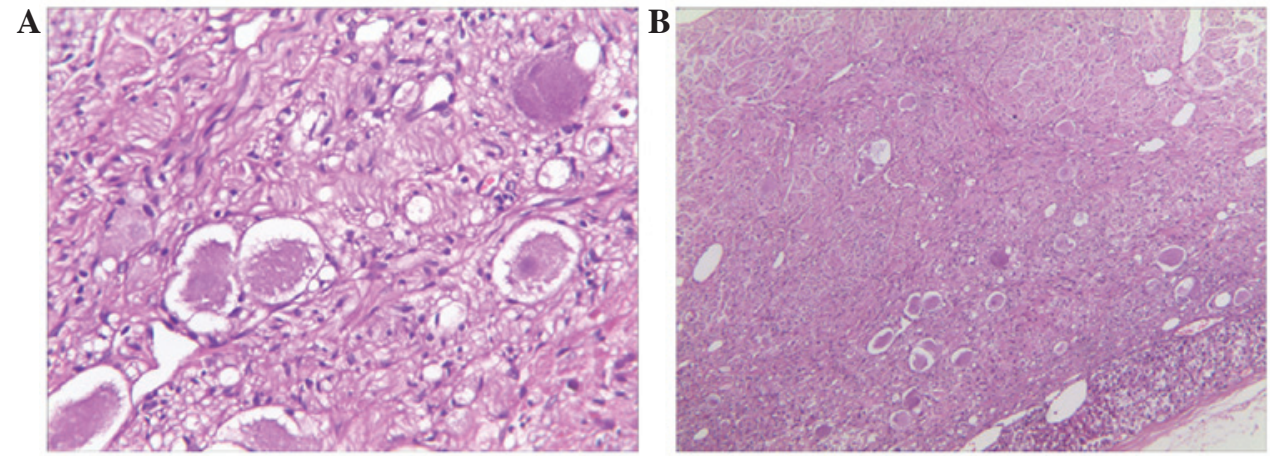

Figure 2. (A) Tumor exhibiting multiple mature ganglion cells in a Schwan cell-dominant stroma with focal lymphocytic infiltration (hematoxylin and eosin stain; magnification, x100). (B) Tumor revealing scattered mature ganglionic cells in a background of spindle-shaped Schwann cells (hematoxylin and eosin stain, magnification, $\mathrm{x} 400$ ).

A

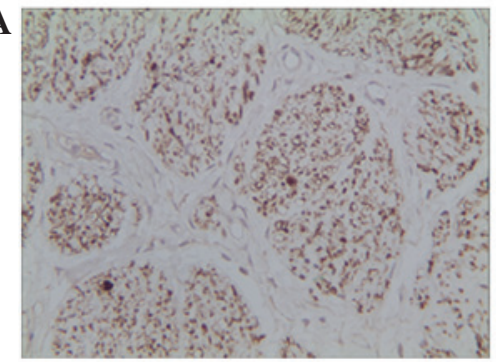

D

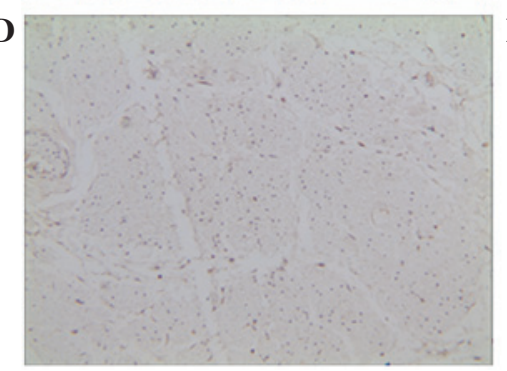

B

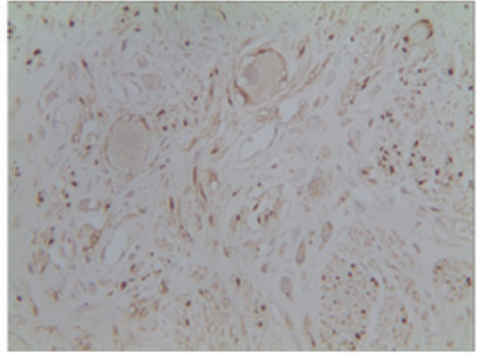

$\mathbf{E}$

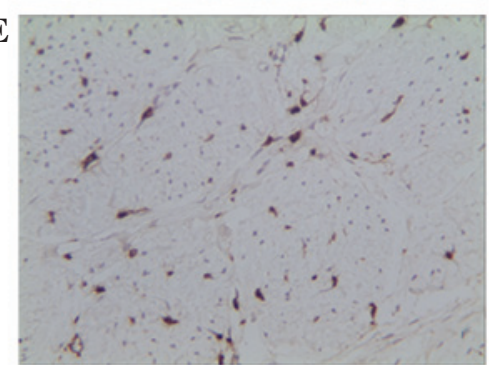

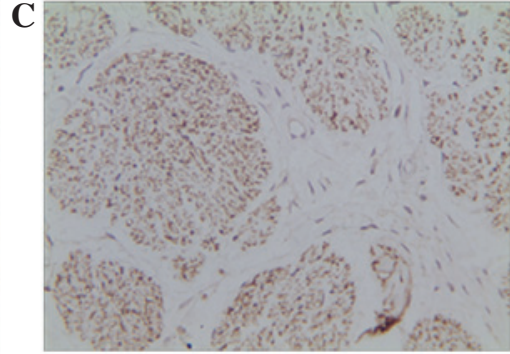

$\mathbf{F}$

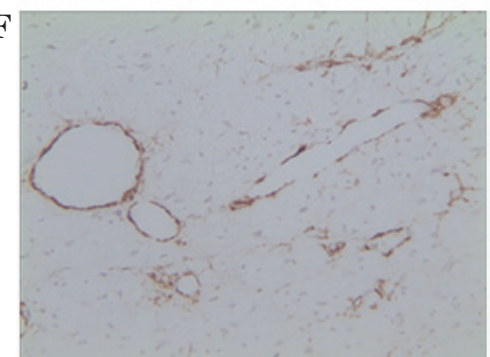

Figure 3. Immunohistochemical analysis revealing the positive expression of (A) neuron-specific enolase, (B) S-100 and (C) cluster of differentiation 56, and the negative expression of (D) cytokeratin, (E) leukocyte common antigen and (F) smooth muscle actin. Magnification, $\mathrm{x} 400$.

distance to neighboring organs or blood vessels. Open surgery is performed in patients with blood loss of $>800 \mathrm{ml}$ and a violent fluctuation in intra-operative blood pressure (4). Due to its benign nature, AGNs rarely metastasize to the regional lymph nodes or distant organs (29), and recurrence is rare following surgical resection (4). For this reason, unnecessary wide excisions should be avoided. Distinguishing between GNs that secrete catecholamines and other neurogenic 
tumors and pheochromocytomas can be challenging. Therefore, if patients present with a high level of catecholamines prior to surgery, a diagnosis of pheochromocytoma should be considered.

In conclusion, forming a pre-operative diagnosis of GN can be a formidable task, particularly in asymptomatic cases. Therefore, early detection, diagnosis and treatment are extremely important. It is common for AGNs to be misdiagnosed, therefore, careful evaluations and surgical treatments are required. Diagnosis can be extremely challenging, and can only be achieved by means of histological evaluation. However, the prognoses of AGNs are usually favorable, and recurrence is rare following surgical resection.

\section{References}

1. Zografos GN, Kothonidis K, Ageli C, et al: Laparoscopic resection of large adrenal ganglioneuroma. JSLS 11: 487-492, 2007.

2. Radin R, David CL, Goldfarb H and Francis IR: Adrenal and extra-adrenal retroperitoneal ganglioneuroma: imaging findings in 13 adults. Radiology 202: 703-707, 1997.

3. Chang CY, Hsieh YL, Hung GY, et al: Ganglioneuroma presenting as an asymptomatic huge posterior mediastinal and retroperitoneal tumor. J Chin Med Assoc 66: 370-374, 2003.

4. Kouriefs C, Leris AC, Mokbel K, et al: Abdominal ganglioneuromas in adults. Urol Int 71: 110-113, 2003.

5. Ichikawa T, Koyama A, Fujimoto $\mathrm{H}$, et al: Retroperitoneal ganglioneuroma extending across the midline: MR features. Clin Imaging 17: 19-21, 1993.

6. Rha SE, Byun JY, Jung SE, et al: Neurogenic tumors in the abdomen: tumor types and imaging characteristics. Radiographics 23: 29-43, 2003.

7. Lonergan GJ, Schwab CM, Suarez ES and Carlson CL: Neuroblastoma, ganglioneuroblastoma, and ganglioneuroma: radiologic-pathologic correlation. Radiographics 22: 911-934 2002.

8. Li L, Shao J, Gu J, et al: Adrenal ganglioneuromas: experience from a retrospective study in a Chinese population. Urol J 11 : 1485-1490, 2014.

9. Erem C, Ucuncu O, Nuhoglu I, et al: Adrenal ganglioneuroma: report of a new case. Endocrine 35: 293-296, 2009.

10. Ambros IM, Zellner A, Roald B, et al: Role of ploidy, chromosome 1p, and Schwann cells in the maturation of neuroblastoma. N Engl J Med 334: 1505-1511, 1996.

11. Domanski HA: Fine needle aspiration of ganglioneuroma. Diagn Cytopathol 32: 363-366, 2005.
12. Pillinger $\mathrm{SH}$, Bambach $\mathrm{CP}$ and Sidhu S: Laparoscopic adrenalectomy: a 6-year experience of 59 cases. ANZ J Surg 72: 467-470, 2002.

13. Tischler AS: Divergent differentiation in neuroendocrine tumors of the adrenal gland. Semin Diagn Pathol 17: 120-126, 2000.

14. Juarez D, Brown RW, Ostrowski M, et al: Pheochromocytoma associated with neuroendocrine carcinoma. A new type of composite pheochromocytoma. Arch Pathol Lab Med 123: 1274-1279, 1999.

15. Chen CL, Huang ST, Chang PL and Ng KF: Adrenal ganglioneuroma: report of five cases. Chang Gung Med J 23: 550-554, 2000.

16. Geoerger B, Hero B, Harms D, et al: Metabolic activity and clinical features of primary ganglioneuromas. Cancer 91: 1905-1013, 2001.

17. Watanabe T, Noshiro T, Kusakari T, et al: Two cases of pheochromocytoma diagnosed histopathologically as mixed neuroendocrine-neural tumour. Intern Med 34: 683-687, 1995.

18. Yamaguchi K, Hara I, Takeda M, et al: Two cases of ganglioneuroma. Urology 67: e1-e4, 2006.

19. Joshi VV and Silverman JF: Pathology of neuroblastic tumors. Semin Diagn Pathol 11: 107-117, 1994.

20. Rao RN, Singla N and Yadav K: Composite pheochromocytoma-ganglioneuroma of the adrenal gland: A case report with immunohistochemical study. Urol Ann 5: 115-118, 2013.

21. Dubois C, Jankowski A, Gay-Jeune C, et al: Imaging of adrenal ganglioneuroma: a case report. J Radiol 86: 659-662, 2005 (In French).

22. Shi BB, Li HZ, Chen C, et al: Differential diagnosis and laparoscopic treatment of adrenal pheochromocytoma and ganglioneuroma. Chin Med J (Engl) 122: 1790-1793, 2009.

23. Elder EE, Edler G and Larsson C: Pheochromocytoma and functional paraganglioma syndrome: no longer the $10 \%$ tumor. J Surg Oncol 89: 193-201, 2005.

24. Manger WM and Eisenhofer G: Pheochromocytoma: diagnosis and management update. Curr Hypertens Rep 6: 477-484, 2004.

25. Kasperlik-Zaluska AA, Roslonowska E, Slowinska- Srzednicka J, et al: 1,111 patients with adrenal incidentalomas observed at a single endocrinological center: incidence of chromaffin tumors. Ann N Y Acad Sci 1073: 38-46, 2006.

26. Maweja S, Materne R, Detrembleur N, et al: Adrenal ganglioneuroma. A neoplasia to exclude in patients with adrenal incidentaloma. Acta Chir Belg 107: 670-674, 2007.

27. Zografos GN, Markou A, Ageli C, et al: Laparoscopic surgery for adrenal tumors. A retrospective analysis. Hormones (Athens) 5: 52-56, 2006.

28. Tsuru N, Ushiyama T and Suzuki K: Laparoscopic adrenalectomy for primary and secondary malignant adrenal tumors. J Endourol 19: 702-709, 2005.

29. Srinivasan R, Koliyadan KS, Krishnand G and Bhat SS: Retroperitoneal ganglioneuroma with lymph node metastasis: a case report. Indian J Pathol Microbiol 50: 32-35, 2007. 